\title{
Constructing a Tandem Mass Spectral Library for Forensic Ricin Identification
}

\section{Supplementary Materials}

Isabelle O'Bryon ${ }^{1}$, Abigail E. Tucker ${ }^{1}$, Brooke L.D. Kaiser ${ }^{1}$, Karen L. Wahl ${ }^{1}$, and Eric D. Merkley ${ }^{1^{*}}$

${ }^{1}$ Chemical and Biological Signature Sciences Group, Pacific Northwest National Laboratory, Richland, Washington

*Corresponding author: eric.merkley@pnnl.gov

\section{List of Figures}

S1 Venn diagrams of shared ricin PSMs and peptides from database searches on ricin standards

S2 Unique ricin peptides present in the spectral libraries.

S3 Variation in the number of ricin PSMs identified across biological and technical replicates

S4 Venn diagram of shared ricin PSMs and peptides from 52 crude castor seed extracts with MS-GF+, Sequest with Percolator, and SpectraST

\section{List of Tables}

S1 Crude castor seed sample list of biological and technical replicates

Table_S1_Crude_Castor_Dataset_Metadata.xIsx

S2 All ricin peptides present in crude castor seed sample from all search methods

Table_S2_Ricin_Peptides_per_file_per_search.txt 
Figure S1a. Shared ricin peptide-spectrum matches from database searches using Sequest with Percolator, Mascot with Percolator, PeaksDB, and MS-GF+ to search the three RKI ricin replicate samples. The majority of PSMs were identified by all database search tools. Only PSMs that were identified by at least two search tools or PSMs that had exceptionally good MS-GF+ SpecE scores were used to create the spectral libraries.

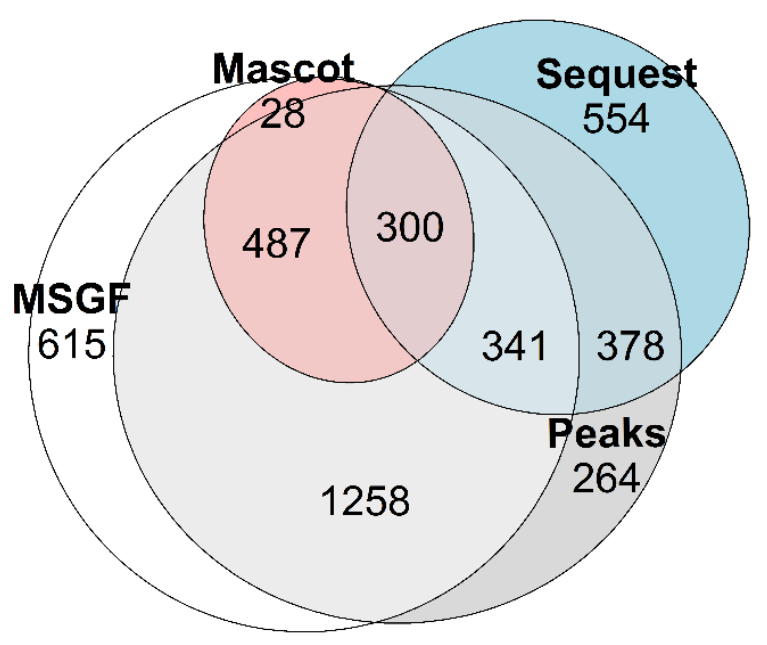


Figure S1.b. Shared ricin peptides from using Sequest with Percolator, Mascot with Percolator, PeaksDB, and MS-GF+ to search the three RKI ricin replicate samples. Mascot was unsuccessful in identifying many of the ricin peptides that the other three database search tools identified.

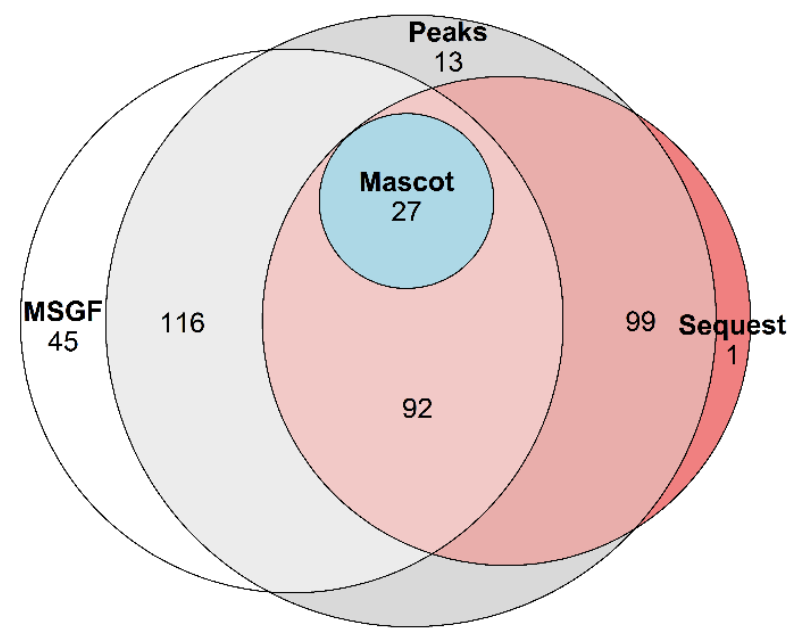


Figure S2a. Unique ricin chain A peptides present in the spectral libraries generated from RKI and Vector ricin samples.

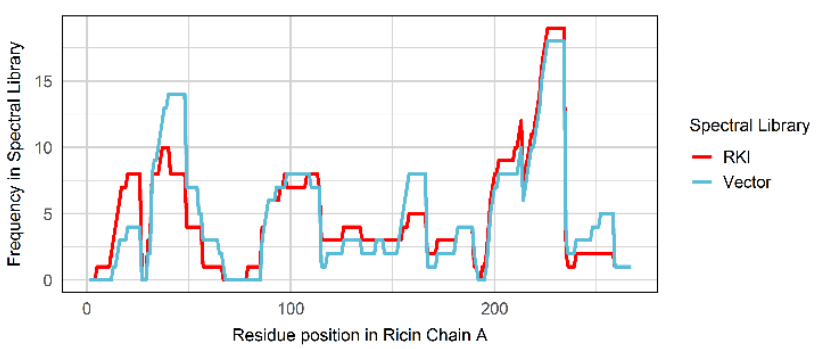


Figure S2b. Ricin chain B peptides present in the spectral libraries generated from RKI and Vector ricin samples. Locations where neither spectral library had coverage indicate areas of ricin chain B that do not contain tryptic cut sites, and therefore peptides from these regions could not be included in the spectral libraries.

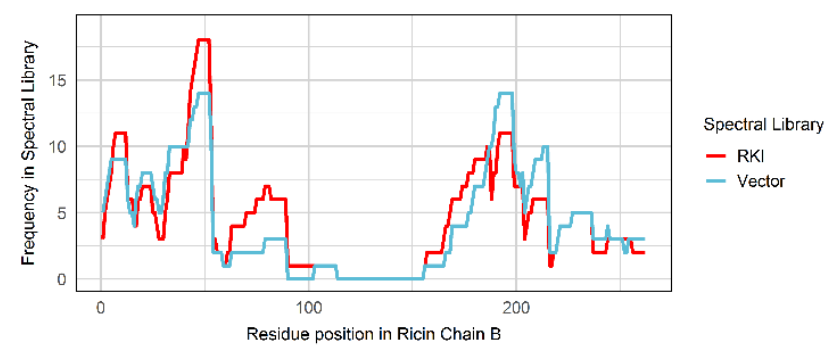


Figure S3a. Variation in the number of ricin PSMs identified across biological and technical replicates using Sequest to search the 52 crude castor seed extracts. Search results were filtered to $1 \%$ FDR.
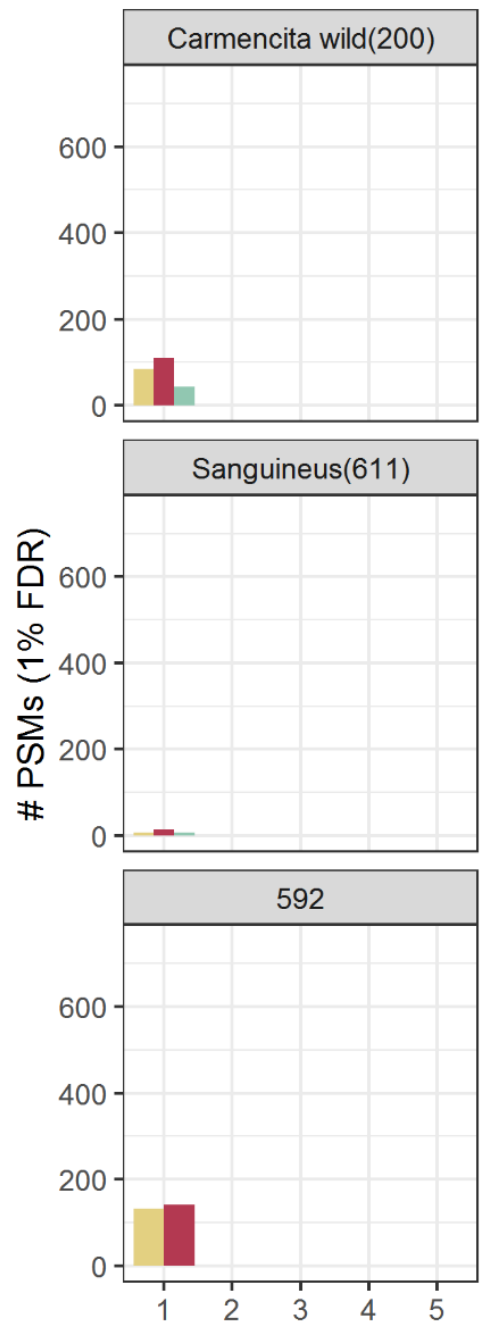
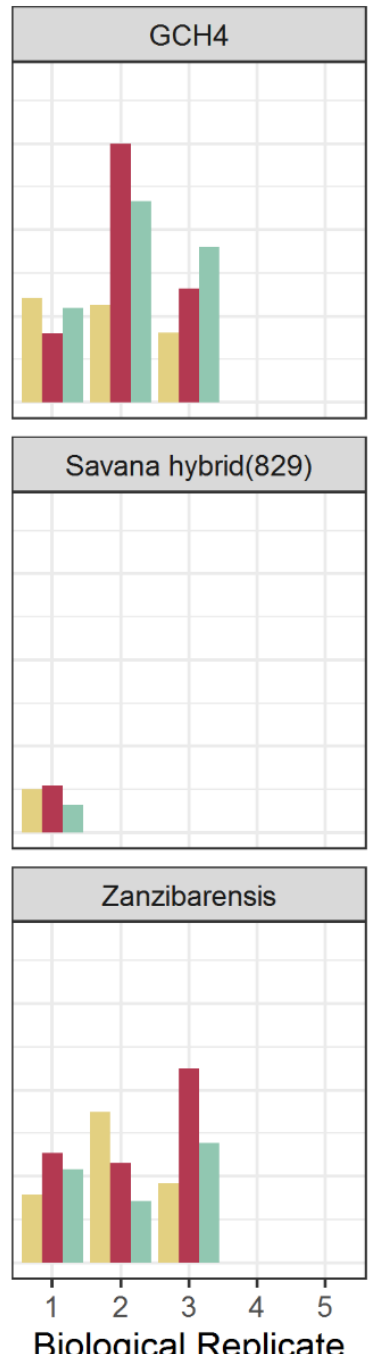
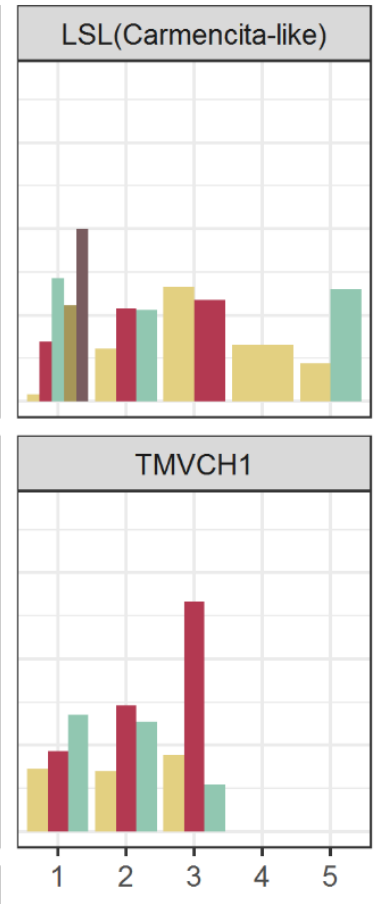

Technical Replicate

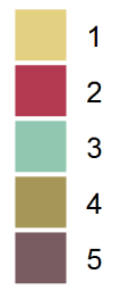


Figure S3b. Variation in the number of ricin SSMs identified across biological and technical replicates using the Vector spectral library to search the 52 crude castor seed extracts using SpectraST. Search results were filtered to $1 \%$ FDR.
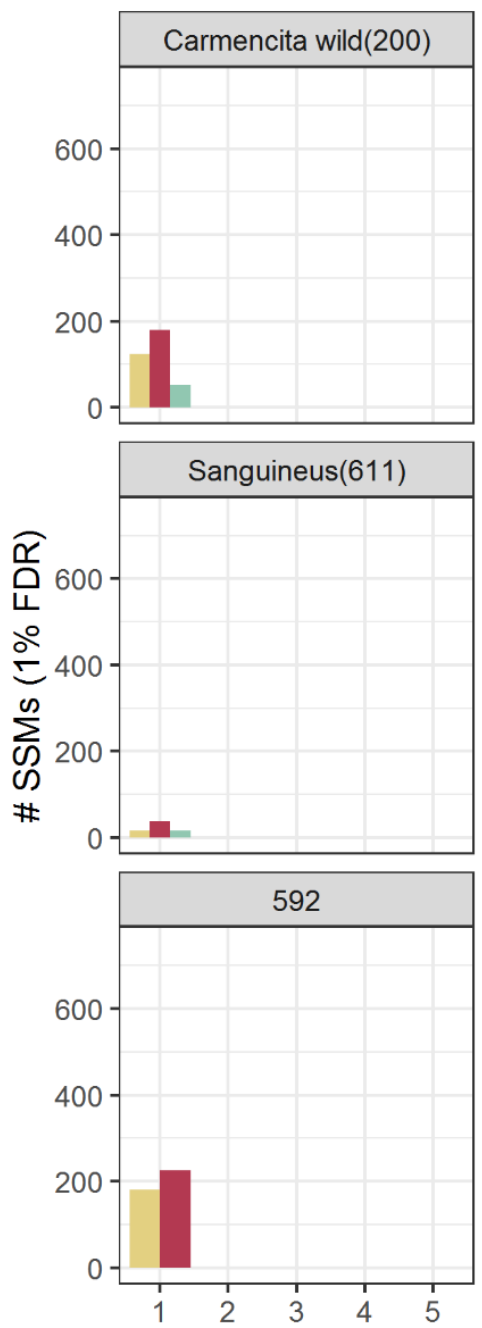

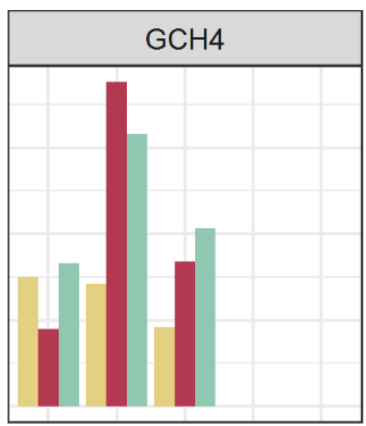

Savana hybrid(829)

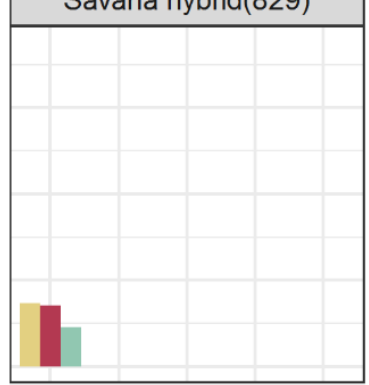

Zanzibarensis

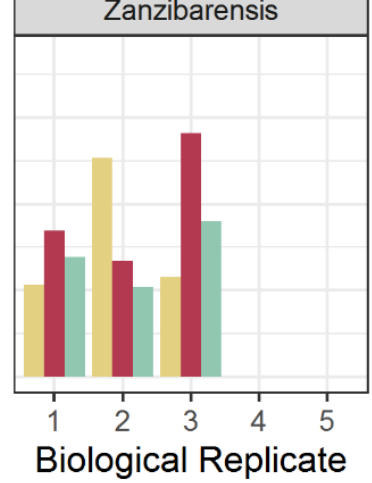

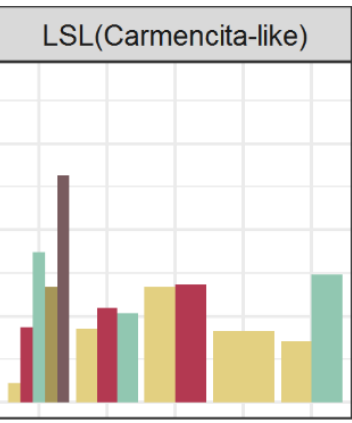

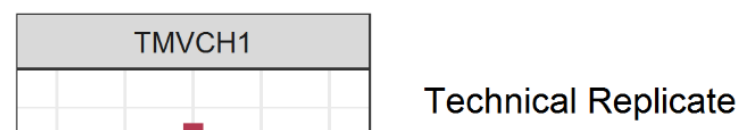

1

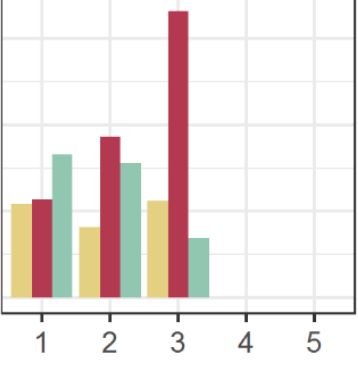

2
3
4
5 
Figure S3c. Variation in the number of ricin PSMs identified across biological and technical replicates using MS-GF+ to search the 52 crude castor seed extracts. Search results were filtered to $1 \%$ FDR.
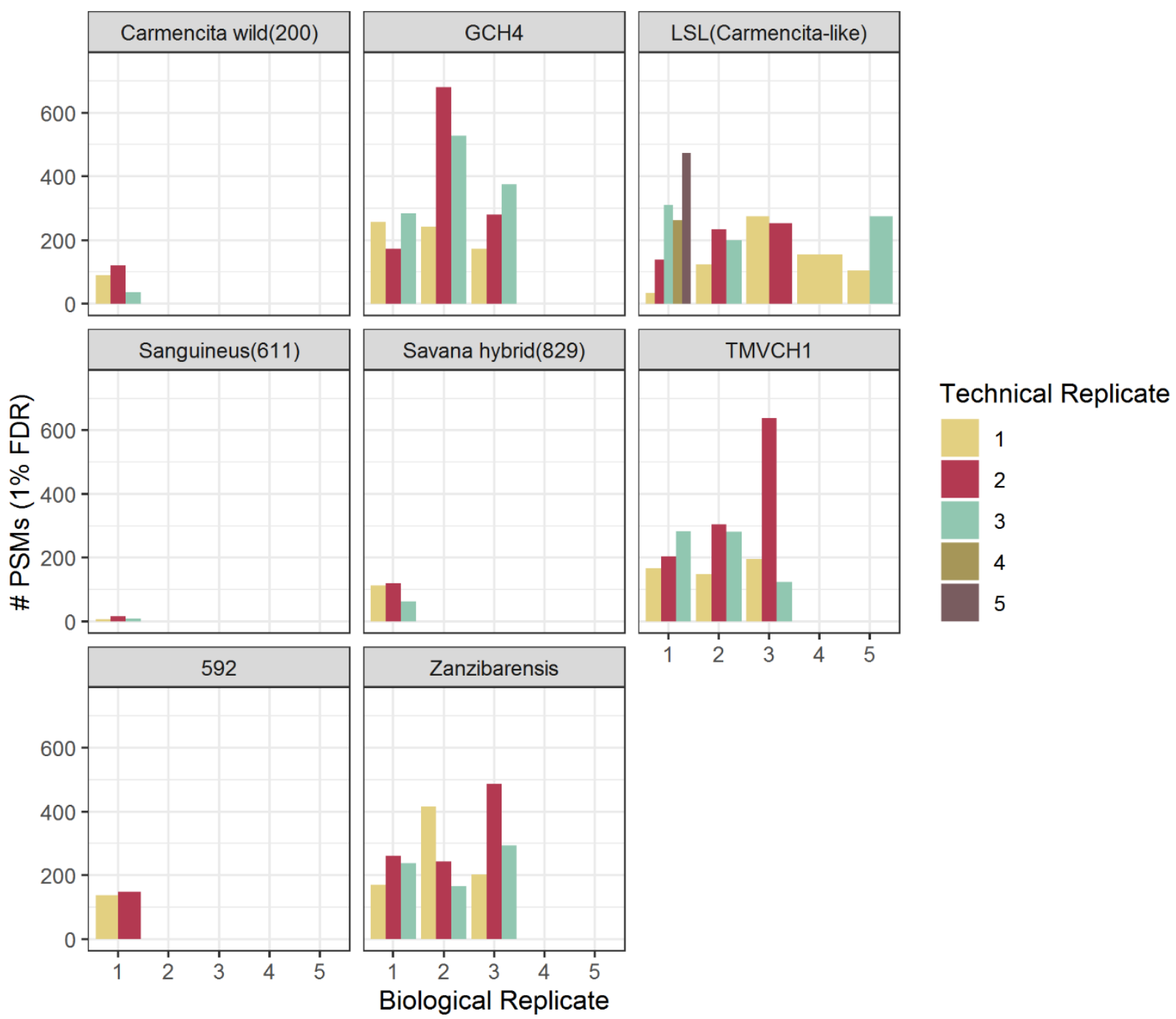
Figure S4a. Venn diagram of the number of shared ricin spectral identifications from searching 52 crude castor seed extracts with MS-GF+, Sequest with Percolator, and SpectraST using the RKI and Vector spectral libraries.

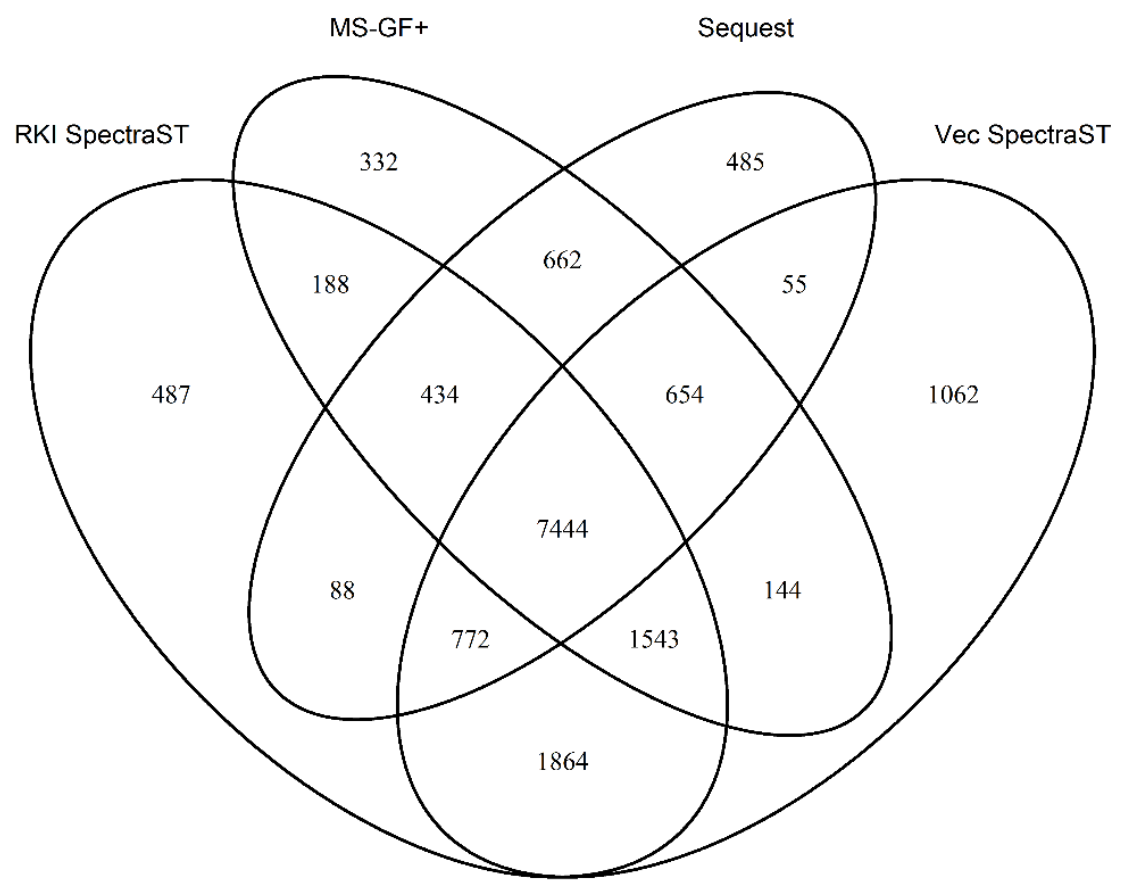


Figure S4b. Venn diagram of the number of shared ricin peptides from searching 52 crude castor seed extracts with MS-GF+, Sequest with Percolator, and SpectraST using the RKI and Vector spectral libraries. See Supplemental Table 1 for the specific peptides found in each dataset by each search method.

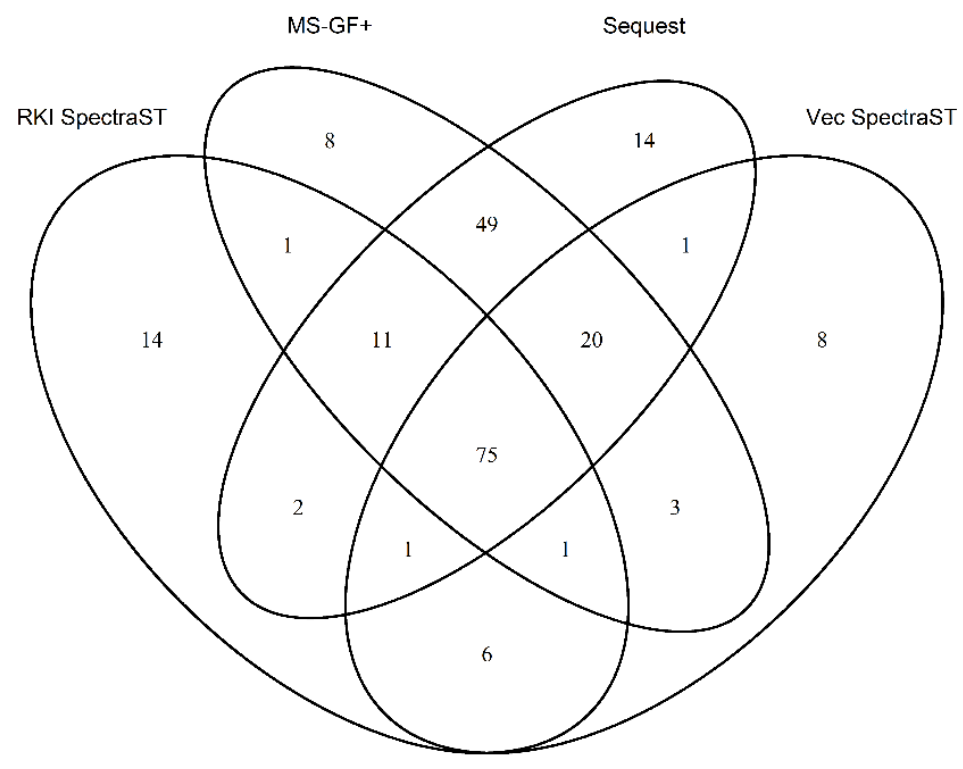

